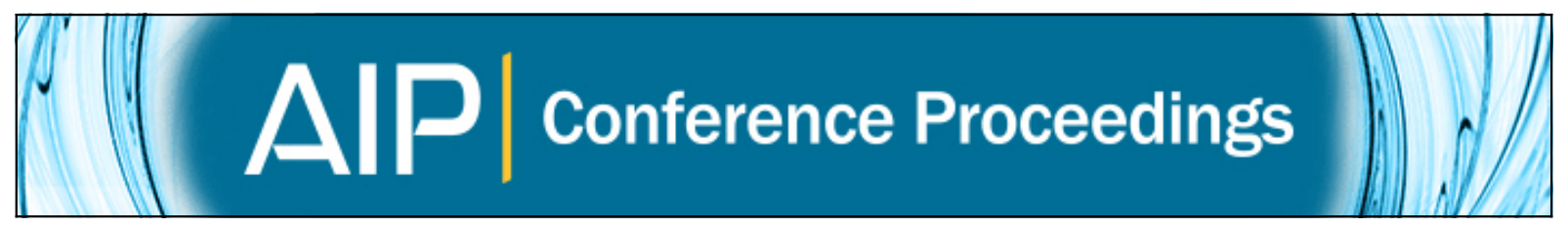

\title{
Evaluation of Constitutive Models for Springback Prediction in U-draw/bending of DP and TRIP Steel Sheets
}

Jeong Yeon Lee, Myoung-Gyu Lee, and Frédéric Barlat

Citation: AIP Conference Proceedings 1383, 571 (2011); doi: 10.1063/1.3623659

View online: http://dx.doi.org/10.1063/1.3623659

View Table of Contents: http://scitation.aip.org/content/aip/proceeding/aipcp/1383?ver=pdfcov

Published by the AIP Publishing

\section{Articles you may be interested in}

A Shop Floor Approach to Springback Prediction

AIP Conf. Proc. 1383, 549 (2011); 10.1063/1.3623656

Draw-Bending Analysis of a Cold Rolled DP980 Steel Sheet

AIP Conf. Proc. 1353, 1405 (2011); 10.1063/1.3589713

New Modelling of Localized Necking in Sheet Metal Stretching

AIP Conf. Proc. 1315, 401 (2011); 10.1063/1.3552478

A Method of Springback Prediction and Tool Shape Compensation for Multi-curvature Sheet Metal Bending AIP Conf. Proc. 1252, 868 (2010); 10.1063/1.3457647

Experimental and numerical study of springback in V-die bending of copper sheets

AIP Conf. Proc. 712, 905 (2004); 10.1063/1.1766642 


\title{
Evaluation of Constitutive Models for Springback Prediction in U-draw/bending of DP and TRIP Steel Sheets
}

\author{
Jeong Yeon Lee, Myoung-Gyu Lee and Frédéric Barlat
}

\author{
Graduate Institute of Ferrous Technology (GIFT), Pohang University of Science and Technology (POSTECH), \\ San 31, Hyoja-dong, Nam-gu, Pohang, 790-784, Republic of Korea
}

\begin{abstract}
U-draw/bending experiments and simulations were performed to investigate the characteristics of springback in sheet metals. The finite element method is frequently used for the simulation of springback, but the predictions are strongly influenced by the constitutive models such as yield criteria and hardening laws. In the present study, springback of DP and TRIP steel sheets after U-draw/bending was predicted with a finite element analysis. Various yield functions were considered, namely, the isotropic von Mises and anisotropic Hill models. For strain hardening, isotropic, non-linear kinematic and combined isotropic-kinematic hardening models were considered. In order to characterize the isotropic hardening behavior, both uniaxial and balanced biaxial (hydraulic bulge) tension tests were carried out. For the characterization of the combined isotropic-kinematic hardening model, forward-reverse simple shear tests were conducted. The springback predictions were greatly influenced by the choice of the hardening model but slightly affected by the choice of the yield criterion. The kinematic hardening and combined isotropic-kinematic hardening models provided satisfactory predictions for DP590 and TRIP590 sheets, respectively. Due to an extended measurable strain range, the use of the flow curves from the hydraulic bulge test led to more reliable results than those of uniaxial tension.
\end{abstract}

Keywords: Springback, U-draw/bending, Finite element simulation, AHSS, Kinematic hardening model, Hydraulic bulge test, Simple shear test

PACS: $62.20 . \mathrm{fq}$

\section{INTRODUCTION}

In sheet metal forming processes, springback is the elastically-driven shape change, which is frequently observed after the material is unloaded. In general, higher residual stresses and lower Young's moduli increase the range of elastic deformation after forming and cause larger springback. Since the automotive industry attempts to utilize Advanced High Strength Steels (AHSS) for manufacturing their products, springback is becoming a significant issue. Finite Element (FE) simulations provide a cost-effective and reliable way of springback predictions for complex geometries and advanced constitutive models. In FE simulations of springback, the constitutive model strongly affects the accuracy of the result. Therefore, the influence of the constitutive models on simulation results has been a common interest in many related studies [1-3]. The role of hardening is particularly significant when the material experiences forward-reverse loading during forming [1,3].

In the present study, U-draw/bending (Numisheet'93 benchmark problem [4]) simulations were conducted to predict springback for two AHSS, a dual-phase (DP) steel and a transformation induced plasticity (TRIP) steel, both with a nominal tensile strength of $590 \mathrm{MPa}$. The aim is to assess the influence of the constitutive model on springback simulations. A constitutive model consists of a yield function and its associated flow rule, and a hardening law. In this work, the von Mises and Hill 1948 yield functions [5] were associated with isotropic, Chaboche-type nonlinear kinematic [6] and combined isotropic-kinematic hardening models [6]. The stress-strain behavior was characterized in two different ways, namely:

(1) Balanced biaxial tension flow curve determined from the hydraulic bulge test as an alternative to the uniaxial tension flow curve to characterize isotropic hardening.

(2) Forward-reverse simple shear curves in order to characterize the combined isotropic-kinematic hardening model. 


\section{EXPERIMENTS}

Two grades of advanced high strength steel sheets, DP590 and TRIP590 (both $1.2 \mathrm{~mm}$ thick) produced by POSCO, were selected for this study. Mechanical tests were conducted in order to characterize the stress-strain behavior and U-draw/bending tests were carried out to assess springback as described below. All tests were duplicated in order to confirm the reproducibility.

\section{Uniaxial Tensile Test}

Uniaxial tension tests were conducted with the Zwick/Roell tensile testing machine on ASTM E8 standard specimens for three loading directions, $0^{\circ}, 45^{\circ}$ and $90^{\circ}$ from the rolling direction (RD). Two mechanical extensometers were attached to the specimen during the test in order to measure the gauge length elongation and the gauge width reduction. The crosshead strain rate was controlled to be $1 \times 10^{-3} / \mathrm{s}$ for all tests. The resulting mechanical properties such as Young's modulus $(\mathrm{E})$, yield stress $\left(\sigma_{\theta}\right)$ and r-value (or plastic strain ratio, $\mathrm{r}_{\theta}$ ) are shown in Table 1 .

\section{Hydraulic Bulge Test}

Hydraulic bulge tests were conducted with the Erichsen bulge/FLC tester model 161. The velocity of the punch, which compresses the hydraulic oil, was set to $12 \mathrm{~mm} / \mathrm{min}$. After the tests, it was confirmed that this velocity resulted in a thickness strain rate of about $3 \times 10^{-3} / \mathrm{s}$. The oil pressure was recorded by a pressure sensor installed in the machine. The oil temperature was maintained between $15{ }^{\circ} \mathrm{C}$ and $35{ }^{\circ} \mathrm{C}$. A mechanical measurement device was placed on the top of the specimen allowing in-plane elongation and curvature measurements using an extensometer and a LVDT (Linear Variable Differential Transformer), respectively. From these measurements, the membrane stress and thickness strain were calculated as described in the literature [7]. The yield stress in balanced biaxial tension $\left(\sigma_{\mathrm{b}}\right)$ was calculated based on the work-equivalence principle by comparing the bulge and uniaxial tension flow curves. The averaged value of flow stress ratio $\sigma_{\mathrm{b}} / \sigma_{0}$ is listed in Table 1 .

\section{Forward-reverse Simple Shear Test}

A device which imposes deformation to a rectangular sample from the parallel displacement of two rigid grips [8] was used for the simple shear tests. The specimens were cut into rectangular shapes of $50 \mathrm{~mm}$ (in the RD) $\times 10 \mathrm{~mm}$ (in the transverse direction, TD) and inserted into the grips of the simple shear device. An appropriate amount of torque, $28 \mathrm{Nm}$, was applied through a set of screws on the grips in order to prevent sliding. The constant grip velocity was set to give a shear strain rate of $2 \times 10^{-3} / \mathrm{s}$ to the sample. The axial force signal was recorded from the load cell and the displacement of the grip was measured by an extensometer. This displacement was assumed to be suitable to calculate the shear strain and to determine the simple shear flow curves. The shear axis was always in the RD. Forward-reverse simple shear tests were conducted for three pre-strains $(5 \%, 10 \%$ and $15 \%$ engineering shear strains) in addition to monotonic simple shear experiments.

\section{U-draw/bending Test}

U-draw/bending tests were performed with a forming machine developed in POSCO. The tool geometry follows the Numisheet'93 benchmark problem [4] and is illustrated in Figure 1(a). The material was cut into rectangular shape specimens with the dimension of $350 \mathrm{~mm} \times 45 \mathrm{~mm}$ (length in the $\mathrm{RD} \times$ width in the TD). A lubricant (BW90EG) was applied on both sides of the specimens. It was measured that this lubricant gives a friction coefficient about $\mu=0.14$. The punch stroke of the U-draw/bending test was fixed to $70 \mathrm{~mm}$. A blank holding force of $25.7 \mathrm{kN}$ was applied to constrain plastic flow.

TABLE 1. Mechanical properties of DP590 and TRIP590 sheets.

\begin{tabular}{ccccccccc}
\hline & $\mathrm{E}[\mathrm{GPa}]$ & $\sigma_{0}[\mathrm{MPa}]$ & $\sigma_{45}[\mathrm{MPa}]$ & $\sigma_{90}[\mathrm{MPa}]$ & $\mathrm{r}_{0}$ & $\mathrm{r}_{45}$ & $\mathrm{r}_{90}$ & $\sigma_{\mathrm{b}} / \sigma_{0}$ \\
\hline DP590 & 191 & 411 & 406 & 391 & 0.91 & 0.84 & 1.12 & 1.02 \\
TRIP590 & 189 & 432 & 442 & 421 & 1.34 & 1.16 & 1.30 & 1.06 \\
\hline
\end{tabular}




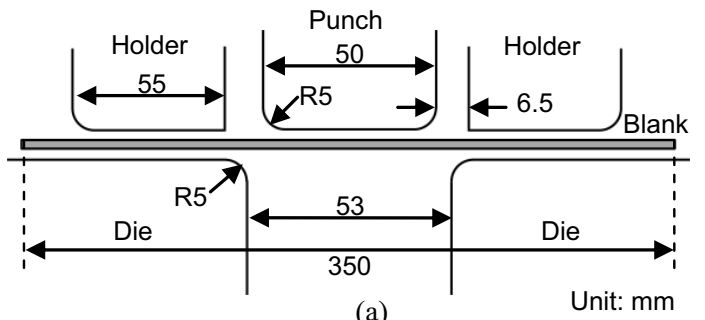

(a)

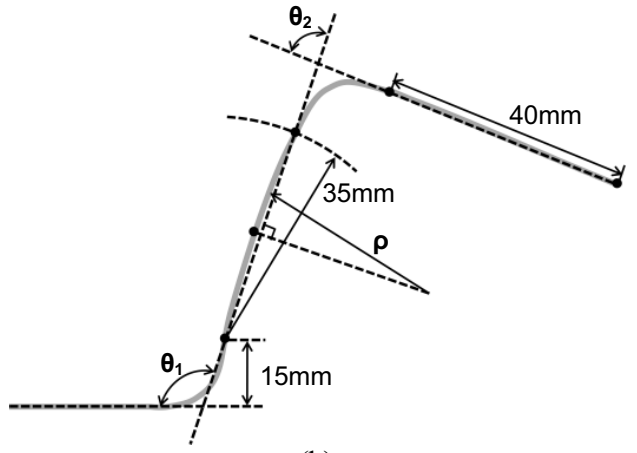

(b)

FIGURE 1. (a) Geometry of U-draw/bending tool and (b) the measurement of springback angles and sidewall curl radius.

After the tests, the edges of the specimens were scanned to a file by 3D digitizing system (Surveyor DS-4060, manufactured by Laser Design Inc.). Then, the springback angles and sidewall curl radii were measured according to the definitions illustrated in Figure 1(b).

\section{CONSTITUTIVE MODELS}

\section{Yield Criteria}

In plane stress, the anisotropic Hill 1948 yield function [5] is expressed as

$$
F \sigma_{y y}^{2}+G \sigma_{x x}^{2}+H\left(\sigma_{x x}-\sigma_{y y}\right)^{2}+2 N \sigma_{x y}^{2}=\bar{\sigma}^{2} .
$$

Here, $\mathrm{x}$ and y correspond to the RD and TD, respectively. $\bar{\sigma}$ represents the effective stress. F, G, H and $\mathrm{N}$ are the yield function coefficients, which are given in Table 2 for DP590 and TRIP590. Note that the coefficients in Table 2 are valid only when RD uniaxial tension is the reference stress state. The yield stress in the RD $\left(\sigma_{0}\right)$ and $r$-values in three different loading directions $\left(\mathrm{r}_{0}, \mathrm{r}_{45}, \mathrm{r}_{90}\right)$, as listed in Table 1, were used to calculate these coefficients.

\section{Hardening Laws}

The hardening behaviors of the two materials under monotonic loading were determined using uniaxial tension and hydraulic bulge tests. These experimental curves were fitted by the Hollomon, $\bar{\sigma}=\mathrm{K}\left(\bar{\varepsilon}^{\mathrm{p}}\right)^{\mathrm{n}}$, and Swift, $\bar{\sigma}=$ $\mathrm{K}\left(\varepsilon_{0}+\bar{\varepsilon}^{\mathrm{p}}\right)^{\mathrm{n}}$, laws as shown in Figure 2. The coefficients for the hardening laws are given in Table 3. The uniaxial flow curve or, alternatively, the balanced biaxial flow curve can be used as a reference for isotropic hardening.

TABLE 2. Coefficients for the Hill1948 yield function (reference: uniaxial tensile stress state).

\begin{tabular}{ccccc}
\hline & $\mathrm{F}$ & $\mathrm{G}$ & $\mathrm{H}$ & $\mathrm{N}$ \\
\hline DP590 & 0.463 & 0.556 & 0.444 & 1.263 \\
TRIP590 & 0.440 & 0.427 & 0.573 & 1.441 \\
\hline
\end{tabular}

TABLE 3. Coefficients of the hardening laws for DP590 (Hollomon) and TRIP590 (Swift).

\begin{tabular}{ccccccc}
\hline & \multicolumn{3}{c}{ Uniaxial tension (RD) } & \multicolumn{3}{c}{ Balanced biaxial tension } \\
\hline & $\mathrm{K}[\mathrm{MPa}]$ & $\varepsilon_{0}$ & $\mathrm{n}$ & $\mathrm{K}[\mathrm{MPa}]$ & $\varepsilon_{0}$ & $\mathrm{n}$ \\
\hline DP590 & 934.19 & - & 0.14 & 976.48 & - & 0.15 \\
TRIP590 & 1113.81 & 0.020 & 0.24 & 1218.20 & 0.016 & 0.24 \\
\hline
\end{tabular}




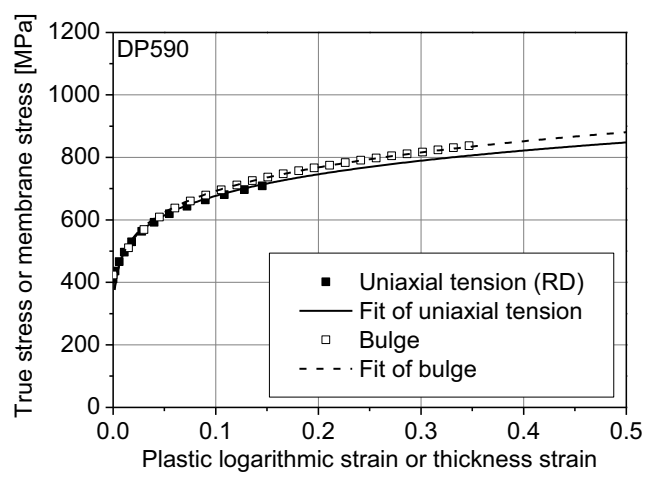

(a)

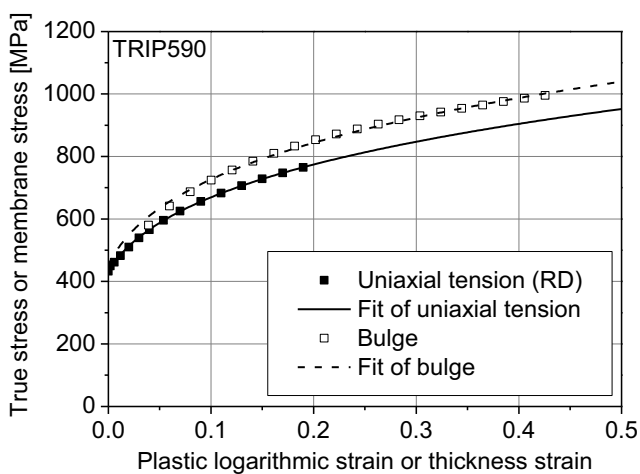

(b)

FIGURE 2. Fitting of hardening curves for (a) DP590 and (b) TRIP590.

According to the Chaboche nonlinear kinematic hardening model [6], the back stress increment is given as a function of effective plastic strain,

$$
\mathrm{d} \boldsymbol{\alpha}=\frac{2}{3} \operatorname{cd} \boldsymbol{\varepsilon}^{\mathrm{p}}-\gamma \boldsymbol{\alpha} \mathrm{d}^{\mathrm{p}}
$$

where $\mathrm{c}$ and $\gamma$ are the material coefficients which are considered constant in this study. In pure kinematic hardening, the size of yield surface does not change and strain hardening is achieved only by the back stress evolution. The parameters for pure kinematic hardening are listed in Table 4.

In the combined isotropic-kinematic hardening model, the yield surface can expand and translate in stress space during plastic deformation. Therefore, an additional evolution rule for isotropic hardening is introduced as,

$$
\bar{\sigma}_{\text {iso }}=\sigma_{0}+\mathrm{Q}\left(1-\exp \left(-\mathrm{b} \cdot \bar{\varepsilon}^{\mathrm{p}}\right)\right)
$$

The subscript 'iso' was given to the effective stress in order to emphasize its contribution to isotropic hardening. This Voce-type isotropic hardening is used, rather than power law hardening, in conjunction with equation (2) for combined isotropic-kinematic hardening. A set of coefficients (see Table 5) for the combined isotropic-kinematic hardening model was determined so that it can reproduce the forward-reverse simple shear curves as shown in Figure 3. Note that a linear combination of several back stress components $\left(\mathrm{d} \boldsymbol{\alpha}=\mathrm{d} \boldsymbol{\alpha}_{1}+\mathrm{d} \boldsymbol{\alpha}_{2}+\cdots\right)$ was used [6].

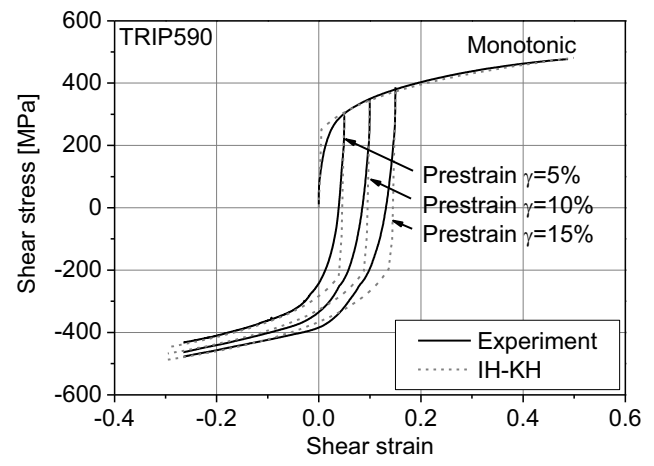

FIGURE 3. Shear stress-strain curves predicted by the combined isotropic-kinematic hardening model (dashed) compared to the experimental curves (solid). 
TABLE 4. Coefficients for pure kinematic hardening model (top: uniaxial tension flow curve, bottom: bulge flow curve as reference).

\begin{tabular}{|c|c|c|c|c|c|c|}
\hline & $\mathrm{c}_{1}[\mathrm{MPa}]$ & $\gamma_{1}$ & $\mathrm{c}_{2}[\mathrm{MPa}]$ & $\gamma_{2}$ & $\mathrm{c}_{3}[\mathrm{MPa}]$ & $\gamma_{3}$ \\
\hline DP590 & 1231 & 2.63 & 5159 & 23.13 & 11511 & 127.80 \\
\hline TRIP590 & 2021 & 2.43 & 4861 & 25.17 & - & - \\
\hline & $\mathrm{c}_{1}[\mathrm{MPa}]$ & $\gamma_{1}$ & $\mathrm{c}_{2}[\mathrm{MPa}]$ & $\gamma_{2}$ & $\mathrm{c}_{3}[\mathrm{MPa}]$ & $\gamma_{3}$ \\
\hline DP590 & 889 & 1.61 & 3173 & 13.57 & 7827 & 51.09 \\
\hline TRIP590 & 757 & 0.36 & 2506 & 6.36 & 4893 & 33.06- \\
\hline
\end{tabular}

TABLE 5. Coefficients for combined isotropic-kinematic hardening model (reference: uniaxial tension flow curve).

\begin{tabular}{ccccccc}
\hline & $\mathrm{Q}[\mathrm{MPa}]$ & $\mathrm{b}$ & $\mathrm{c}_{1}[\mathrm{MPa}]$ & $\gamma_{1}$ & $\mathrm{c}_{2}[\mathrm{MPa}]$ & $\gamma_{2}$ \\
\hline TRIP590 & 1200 & 0.50 & 3101 & 9.83 & 3388 & 47.60 \\
\hline
\end{tabular}

In order to compare the forward and reverse stress-strain behavior from the different hardening models, single element FE simulations were performed for uniaxial tension-compression. The corresponding stress-strain curves are shown in Figure 4.

\section{SPRINGBACK PREDICTIONS}

\section{Finite Element Modeling}

A FE model of the U-draw/bending process was constructed in Abaqus/Standard (implicit code) version 6.8 based on the tool geometry shown in Figure 1(a). Only a quarter of the whole model was considered because of the geometrical and material symmetries. A blank holding force of $6.4 \mathrm{kN}$ was applied on the quarter geometry, and the friction coefficient was $\mu=0.14$. The tools, namely, the punch, die and blank holder, were modeled as analytical rigid surfaces while the blank was meshed with shell elements with reduced integration (S4R). Plane stress conditions were assumed in the quarter model. It was found from a series of parametric study that the use of $1 \mathrm{~mm} \times 1.5 \mathrm{~mm}$ (length $\times$ width) mesh and 9 integration points through the thickness was reliable for the current geometry.

\section{Influence of the Yield Function}

In order to observe the influence of the yield function, springback was predicted with two different models (von Mises and Hill 1948) and isotropic hardening, characterized with the uniaxial tension flow curve. The results are not shown in this paper, but elsewhere [9]. These two yield functions predicted very similar springback for DP590 and TRIP590, which is probably due to the isotropic yielding behavior of these materials.

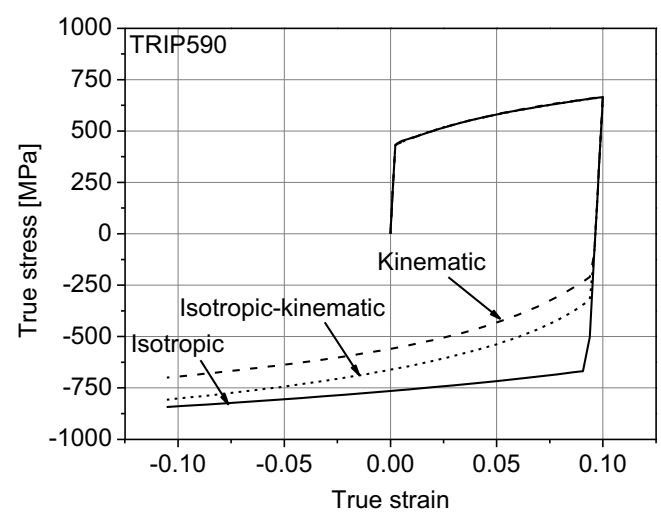

FIGURE 4. Stress-strain curves under tension-compression for different hardening models, obtained from FE simulations. 


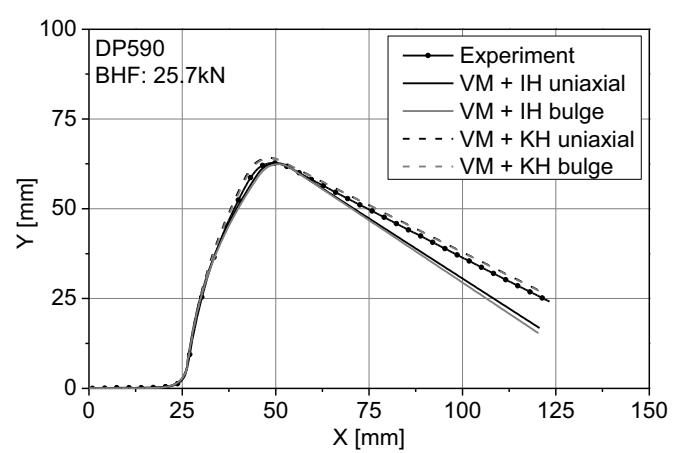

(a)

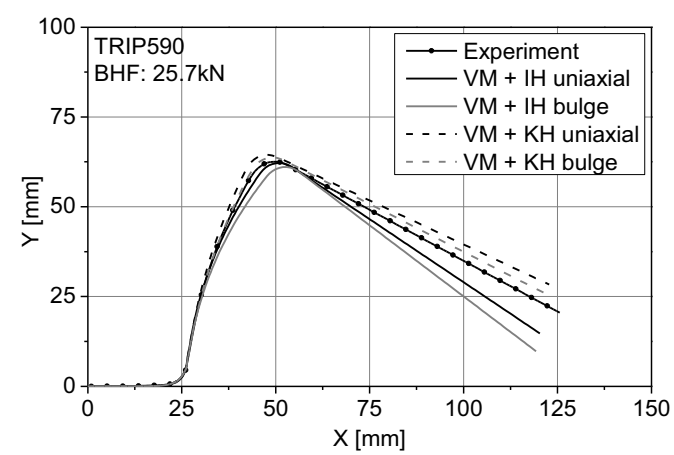

(b)

FIGURE 5. Springback predicted by different hardening models and different reference curves for (a) DP590 and (b) TRIP590.

\section{Influence of the Hardening Laws}

Figure 5 shows springback predictions with two different hardening models, the isotropic and Chaboche kinematic hardening models. Each hardening model is characterized by either the uniaxial tension (indicated as 'uniaxial') or bulge (indicated as 'bulge') flow curves. The von Mises yield criterion was selected for all the simulations shown in Figure 5.

For DP590, the choice of the reference hardening curve ('uniaxial' or 'bulge') seems to have little effect on the springback predictions. This might be related to the similarity of two reference curves shown in Figure 2(a). However, the choice of the reference curve certainly affects the predictions for TRIP590. This is likely due to the fact that the bulge flow curve is much higher than the uniaxial flow curve for TRIP590, Figure 2(b), leading to higher residual stresses. This results in larger predicted springback with the bulge flow curve.

The choice of the hardening model, either isotropic or kinematic, has more influence on springback than the choice of either the yield function or the reference hardening curve. The isotropic hardening model tends to overestimate springback while the kinematic hardening model tends to underestimate it. This is mainly because of the difference in residual stresses obtained through forward-reverse bending in the sidewall region. The kinematic hardening model shows early re-yielding (or Bauschinger effect) which makes the final residual stress much lower than that by the isotropic hardening model. Therefore, a smaller springback is expected with kinematic hardening compared to isotropic hardening.

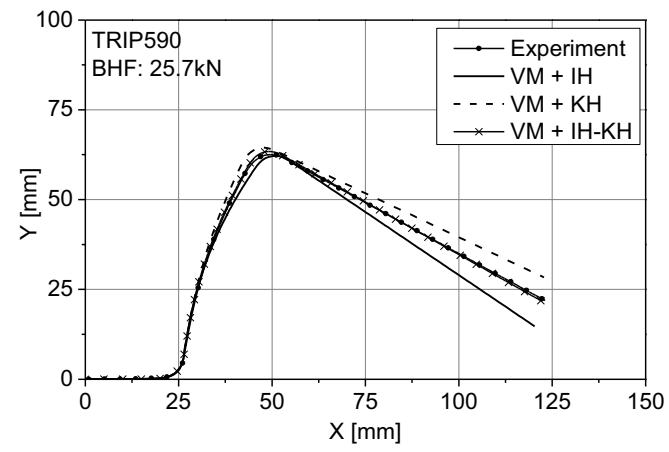

(a)

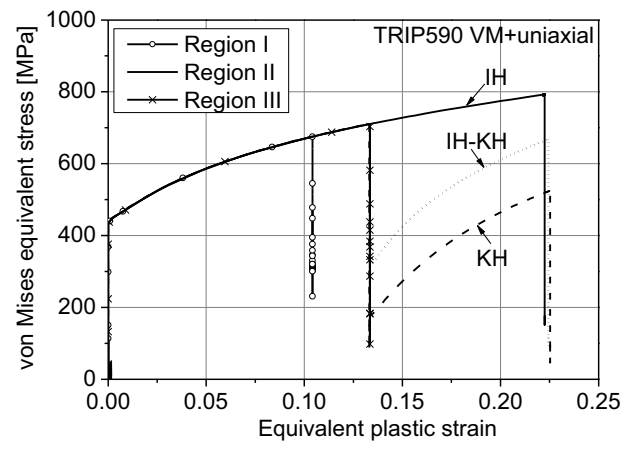

(b)

FIGURE 6. (a) Springback predicted by different hardening models and (b) Von Mises equivalent stress-strain curves obtained from finite elements in Regions I (punch corner), II (sidewall) and III (die corner). 


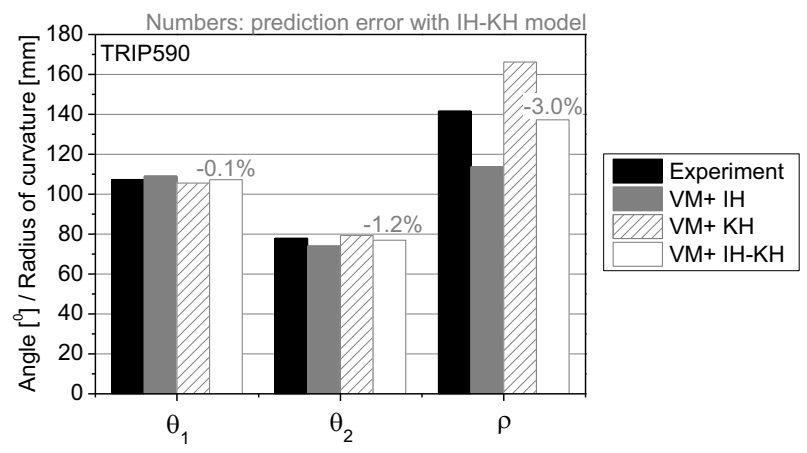

FIGURE 7. Springback angles and radii of curvature predicted by three different hardening models for TRIP590.

In terms of accuracy, springback of DP590 is well predicted by kinematic hardening with roughly a $1 \%$ error for springback angles and a 3\% error for sidewall curvature. For TRIP590, the springback angles are also well predicted by kinematic hardening within an error smaller than $2 \%$. However, the sidewall curvature is not well captured using either the reference behavior from uniaxial tension (17\% error) or bulge ( $9 \%$ error) tests.

Since the prediction of the curl radius was not satisfactory for TRIP590, the combined isotropic-kinematic hardening model was employed for that material. Figure 6(a) shows the springback profiles predicted with three different hardening models, and Figure 7 shows the corresponding springback angles and sidewall curl radii. The von Mises yield criterion and reference hardening curve in uniaxial tension were consistently used for these simulations. It was found that the springback predictions were much improved using the combined isotropickinematic hardening model comparing to isotropic or by kinematic hardening. This result can be explained in terms of residual stresses in the sidewall at the end of forming. The equivalent stress-strain curves were extracted from the finite elements at three regions (I: in contact with punch corner; II: in contact with die corner; III: sidewall) as shown in Figure 6(b). Only monotonic loading occurs in Regions I and III, for which any hardening model leads to the same stress-strain response. If the load is reversed, as in Region II, the kinematic hardening and combined hardening models are characterized by early reverse yielding (or Bauschinger effect), which makes the final residual stress much smaller than that made with isotropic hardening. It is worth noting that the residual stress predicted from combined isotropic-kinematic hardening is between those from isotropic and kinematic hardening. Considering the fact that springback is proportional to the residual stress, the springback obtained from combined hardening is intermediate between the springbacks obtained from the other two models. Since this explanation is valid only in the sidewall region, the differences between the hardening models are significant only for the sidewall curl radius, as shown in Figure 7.

\section{DISCUSSION}

In this study, the materials were characterized using bulge and forward-reverse simple shear flow curves. Based on the experimental and simulated results presented above, a few comments about these characterization methods are given below.

It is common to use the uniaxial tension flow curve to characterize the isotropic hardening behavior. The finite element simulation in Figure 6(b) showed that the plastic equivalent strain during U-draw/bending can exceed $20 \%$. It is difficult to obtain the flow curves up to this level of plastic strain in uniaxial tension tests for some AHSS sheets. For this reason, the uniaxial tension flow curves are extrapolated using power or exponential laws. Generally the hydraulic bulge test provides the flow curve with a much extended strain range. For example, for the DP590 and TRIP590 used in this work, the maximum strains were in the range $35-40 \%$. The use of the bulge flow curve can, therefore, reduce a possible error coming from extrapolations if the hardening curves are similar in both uniaxial tension and balanced biaxial tension such as for DP590 (Figure 2(a)). When the flow curves are quite different like for TRIP590 (Figure 2(b)), there is no clear answer to which flow curve is more suitable for U-draw/bending (which is often regarded as plane strain problem, i.e., between uniaxial and balanced biaxial tension). 
In order to characterize the material with combined isotropic-kinematic hardening, it is necessary to characterize the material behavior under non-monotonic loading. This is usually achieved with uniaxial tension-compression or three-point bending tests $[1,3]$. Uniaxial tension-compression test is a straight-forward procedure to obtain the model parameters [1]. However, the maximum strain achievable in compression is limited due to elastic or plastic buckling. In contrast, the three-point bending test has no such a limitation and the experimental procedure is rather simpler. However, additional FE simulations are necessary for the three-point bending and the model parameters are indirectly determined through numerical iteration schemes [3]. In the forward-reverse simple shear test, as proposed in this research, the maximum strain achieved is much larger than in uniaxial tension-compression. This also requires additional FE simulations for simple shear but simpler than for three-point bending because a single element simulation is sufficient in this case. It was not verified in this work that the forward-reverse simple shear behavior allows a good prediction of forward-reverse bending. However, the model coefficients obtained using forward-reverse simple shear were shown to predict a reasonable uniaxial tension-compression behavior.

\section{CONCLUSIONS}

The springback in the U-draw/bending process was predicted for two AHSS using various yield functions, von Mises and Hill 1948, and hardening laws, isotropic, kinematic and combined isotropic-kinematic hardening models. The mechanical properties of the two AHSS sheets, DP590 and TRIP590, were investigated using uniaxial tension, hydraulic bulge and simple shear tests. The measured properties were used in the different constitutive models in order to simulate springback. A comparative analysis leads to the following conclusions:

(1) For DP590 and TRIP590, the yield criterion has a minor effect on springback while the hardening model plays a major role.

(2) Isotropic hardening tends to overestimate springback while kinematic hardening tends to underestimate it, especially regarding the radius of curvature in the sidewall region. This tendency is directly related to the difference in residual stress levels produced by these models at the end of forming.

(3) The springback predictions also depend on the choice of the reference hardening curve, i.e., uniaxial tension or bulge flow curves, but not as much as the hardening model.

(4) The springback predictions for DP590 were satisfactory within an acceptable error using the kinematic hardening model. In contrast, the predictions for TRIP590 were satisfactory only with the combined isotropickinematic hardening model.

\section{ACKNOWLEDGEMENTS}

This research was supported by POSCO and by WCU (World Class University) program through the National

Research Foundation of Korea funded by the Ministry of Education, Science and Technology (R32-10147). Experiments were conducted with the help of Ms. Le Xu, Mr. Hyun Jin Kim, Mr. Jin Woo Lee and Mr. Seok Nyeon Kim from Materials Mechanics Laboratory, GIFT, POSTECH.

\section{REFERENCES}

1. M. G. Lee, D. Kim, C. Kim, M. L. Wenner, K. Chung, Int. J. Plasticity 21, 915-953 (2005)

2. R. H. Wagoner, J. F. Wang, M. Li, "Springback" in ASM Handbook volume 14B Metal Working: Sheet Forming, ASM, Material Park, OH, 2006, pp. 733-755.

3. P. A. Eggertsen, K. Mattiasson, Int. J. Mech. Sci 51, 547-563 (2009).

4. L. Taylor, J. Cao, A. P. Karafillis, M. C. Boyce, J. Mater. Proc. Technol. 50, 168-179 (1995).

5. R. Hill, Proc. Roy. Soc. Lond. 193, 281-297 (1948).

6. J. L. Chaboche, Int. J. Plasticity 2, 149-188 (1986).

7. M. G. Lee, D. Kim, C. Kim, M. L. Wenner, R. H. Wagoner, K. Chung, Int. J. Plasticity 21, 883-914 (2005).

8. E.F. Rauch, "Non-linear phenomena in materials science II" in Solid State Phenomena volume 23-24, edited by G. Martin, L. Kubin, 1992, pp. 317-334.

9. J. Y. Lee, Master thesis, Pohang University of Science and Technology, 2011. 